\title{
AB1014 THE VALUE OF AN AUTOMATED ULTRASOUND SYSTEM IN THE DETECTION OF SYNOVITIS
}

R. Mueller $^{1}$, M. Gruenke ${ }^{2}$, J. Wendler ${ }^{3}$, F. Schuch ${ }^{3}$, H.-P. Karina ${ }^{4}$, I. Boettger ${ }^{4}$, R. Jakobs ${ }^{5}$, H. Schulze-Koops ${ }^{2}$, J. von Kempis ${ }^{1} .{ }^{1}$ Rheumatology, Kantonsspital St. Gallen, St. Gallen, Switzerland; ${ }^{2}$ Division of Rheumatology, Medizinische Klinik und Poliklinik IV, University of Munich, Munich; ${ }^{3}$ Schwerpunktpraxis Rheumatologie; ${ }^{4}$ Institut für bildgebende Diagnostik und Therapie, BDT - MVZ Träger GmbH, Wetterkreuz 21; ${ }^{5}$ Siemens, Erlangen, Germany

Background: The detection of joint swelling caused by synovitis is important for the diagnosis and assessment of inflammatory arthritis. Ultrasound (US) and MRI have proven to be more sensitive and reliable than physical examination, but a comprehensive examination of affected joints with these techniques is time consuming and expensive. The automated breast volume scanner (ABVS) was developed to acquire serial B-mode pictures of the female breast and these data can be analysed in all three dimensions.

Objectives: To analyse the value of automated grey scale B mode US employing the ABVS system in detecting synovitis of the finger joints compared to manual ultrasound and physical examination, using MRI as gold standard.

Methods: 19 consecutive patients suffering from rheumatoid $(n=15)$ or psoriatic $(n=4)$ arthritis with at least one swollen finger joint were included. Automated and manual US were conducted using the ACUSON S2000 ${ }^{\mathrm{TM}}$. The ABVS transducer was equipped with a linear array used with a frequency of $11 \mathrm{MHz}$. Each automatic sweep of the scanner generated $15.4 \times 16.8 \times 2.5 \mathrm{~cm}$ volume data sets. The system was set to perform an automatic scanning time of $65 \mathrm{~s}$ per scan with a slice thickness of $0.5 \mathrm{~mm}$. The dorsal and palmar side of each hand were scanned separately. Multiplanar reconstruction enabled examination of the images at multiple levels for the presence of synovitis.

Results: Automated US detected 12.0, manual ultrasound 14.2, MRI 13.4, and clinical examination 4.1 swollen joints on average, respectively, per patient. The inter-observer reliability of both assessors for automated and manual US, MRI, and physical examination, was $66.9 \%, 72.7 \%, 95.1 \%$, and $88.9 \%$, respectively. A double assessor detection of joint swelling with MRI was used as gold standard. For the other methods, single observer detection was chosen. $84.3 \%$ of the joints classified as positive on MRI were confirmed by automated ultrasound, $85.5 \%$ on manual US, and 36.0 on physical examination. This translated into a sensitivity of $83.5 \%, 85.5 \%$, and $36.0 \%$ for the three methods, respectively.

Conclusions: Automated US is a simple and time sparing option for the effective detection of synovitis in patients with inflammatory arthritis.

Disclosure of Interest: R. Mueller: None declared, M. Gruenke: None declared, J. Wendler: None declared, F. Schuch: None declared, H.-P. Karina: None declared, I. Boettger: None declared, R. Jakobs Employee of: Siemens, H. Schulze-Koops: None declared, J. von Kempis: None declared

DOI: 10.1136/annrheumdis-2014-eular.2059 